richiamo a Schmitt è possibile anche quando in Oakeshott sembra trattarsi solo del lato 'associativo', 'amichevole', del rapporto; è vero che Schmitt sottolinea il momento conflittuale e dunque il 'nemico' nella relazione, ma, come Schmitt non può sottovalutare i necessari effetti associativi del momento conflittuale, così Oakeshott riconosce certamente molto bene le possibili conseguenze 'polemogene' di ogni vita etica condotta in maniera sostanziale. Del resto, la critica al 'razionalismo' in politica ${ }^{10}$ deve essere intesa come la critica ad un fenomeno storicamente reale che, piaccia o meno, ha informato di sé buona parte della storia dell'Occidente negli ultimi secoli, una forma dell'azione politica, quella della 'fede' 11 , che rappresenta un polo nel pendolo delle vicende umane, ma un polo per l'appunto reale, sul quale ogni tanto è inevitabile che ci si appoggi, anche quando intellettualmente si vorrebbe stare dall'altra parte. È una delle ragioni per le quali annovero questo filosofo dello scetticismo alla tradizione del realismo politico.

\title{
ALLE ORIGINI DELLO SCIENTISMO: BENTHAM
}

\section{di Raimondo Cubeddu*}

Pur essendo considerato uno dei maggiori filosofi politici del Novecento, Oakeshott, come è noto, non aveva una grande considerazione della politica e riteneva anzi che nel corso dei secoli avesse costantemente offerto uno "spettacolo sgradevole"12. Si potrebbe addirittura pensare che se ne occupasse, sia pure nel modo particolare in cui lo faceva, per mettere in guardia dal farsi illusioni su di essa, o forse per

un'assenza alla quale occorre porre rimedio se vogliamo adeguare il suo concetto di societas". Naturalmente, il "noi" della Moufffe è un "noi" nel quale "noi" non ci riconosciamo, pur apprezzando l'onestà intellettuale della stessa Mouffe.

${ }^{10}$ M. OAKeShOTT, Razionalismo in politica, cit.

${ }^{11}$ M. OAKeshott, The Politics of Faith and the Politics of Scepticism, ed. by T. FuLLER, London, Yale University Press, 1996; ed. it. a cura di A. CARRINO, La politica moderna tra scetticismo e fede, Soveria Mannelli, Rubbettino, 2013.

12 M. OAKeShott, La politica moderna tra scetticismo e fede, cit., p. 27.

* Università di Pisa. 
mettere in evidenza i guai che poteva combinare l'idea di migliorare la condizione umana tramite la politica. Soprattutto dopo che essa è diventata "scientifica".

Da questo punto di vista, e rinunciando a fare un sia pur smilzo paragone tra l'idea che altri due giganti della filosofia politica novecentesca, quali Hayek e Leo Strauss, si erano autonomamente fatta delle calamità che sarebbero derivate dall' applicazione alla politica delle finalità, degli ideali e dei metodi di quello che a partire dagli anni Quaranta inizierà a denominarsi scientismo, la prima cosa che questo saggio mostra è una riprova del fatto che la filosofia, e le analisi filosofiche, per quanto possano essere accurate, perspicaci e quant'altro, hanno un impatto sulla realtà e sullo sviluppo sociale che definire imprevedibile è forse poco.

Per certi versi, quindi, il saggio di Oakeshott su Bentham mi ha ricordato quello di Böhm-Bawerk su Marx: La conclusione del sistema marxiano, del 189613. Come Böhm-Bawerk, il quale al termine di un'analisi acuta, pacata, illuminante ma anche devastante, dichiarava di dubitare che idee sul valore così contraddittorie come quelle di Marx avrebbero potuto avere futuro e fortuna, anche Oakeshott si chiede come mai pensieri così sconclusionati come quelli di Bentham abbiano potuto riscuotere tanto successo. Quel che invece stava avvenendo ed avvenne è che ad avere successo sono state le idee di Marx e di Bentham e non le illuminanti analisi di Böhm-Bawerk e di Oakeshott. A riprova del fatto che su ciò che determina il successo di un'idea in realtà sappiamo poco di più del fatto che il loro contenuto di verità può essere (ed in genere è) irrilevante.

Fin dalle prime righe il saggio di Oakeshott è decisamente irriverente nei confronti di Bentham, e nel prosieguo non manca di rimarcare come le sue idee non soltanto fossero assai poco originali ma anche come fossero pure mal formulate. Perché, dunque, ebbero successo?

Il punto di partenza di Oakeshott sono tre libri su Bentham apparsi pochi anni prima: Charles W. Everett, The Education of Jeremy Bentham, e Bentham's Comments on Commentaries ${ }^{14}$, e C.K. Ogden, Ben-

13 Ora ritradotto e pubblicato, con un mio saggio introduttivo, da IBL Libri, Torino, 2020.

${ }^{14}$ Cfr. C.W. Everett (ed. by), Bentham's Comments on the Commentaries, Oxford, Clarendon Press, 1928); e C.W. EvereTt., The Education of Jeremy Bentham, New York, Columbia University Press, 1931. 
tham 's Theory of Legislation ${ }^{15}$, che lo rigenerano presentandolo in una maniera decisamente diversa da quella tradizionale e ponendolo come padre nobile di quella tendenza ad una "politica scientifica" che si stava affermando proprio in quegli anni.

Oakeshott non nega che Bentham sia stato un personaggio importante già ai suoi tempi, ma certamente non ritiene sia stato - come invece si vorrebbe far credere - uno dei più importanti. E infatti, per quanto citi Albert V. Dicey secondo il quale "“l'intera storia della riforma del diritto in Inghilterra è la storia dell'ombra proiettata da un solo uomo: Bentham"' (p. 267), Oakeshott ritiene che neanche in questo ambito l'apporto di Bentham sia stato fondamentale, e che comunque si fondava su presupposti filosofici erronei come quello che fosse possibile arrestare l'evoluzione del diritto.

Dopo essersi soffermato sul rapporto col suo figlioccio John S. Mill, il quale dichiarò di aver trovato in Bentham una religione, sull'immagine che ne avevano i suoi contemporanei, e sulla nuova e diversa immagine di Bentham fornita da Everett e da Ogden - i quali, muovendo da nuove informazioni sulla sua vita trasformano quello che era un sincero figlio del suo tempo: un parente di Claude-A. Helvetius, di Denis Diderot, di Voltaire e di Jean Baptiste d'Alembert, ovvero sostenitore del "dispotismo illuminato" (p. 269), in un pensatore dei tempi moderni e delle relative problematiche - Oakeshott espone il proprio pensiero su Bentham.

A suo avviso Bentham fu una mente indubbiamente ingegnosa dotata di vivacità ma "non di capacità di scegliere" (p. 271), e nella quale comunque mancano una seria prospettiva filosofica e originalità dato che le sue idee provengono sostanzialmente da John Locke, da David Hume, da Ėtienne Bonnot de Condillac e da Helvetius (p. 281). Ciò che induce Oakeshott a sostenere che, per quanto sia stato un iniziatore di molte riforme pratiche che furono molto più apprezzate nel Continente che in Inghilterra, Bentham come i Philosophes, le concepì soltanto a metà e comunque non in maniera tale da estendere le nostre conoscenze sul diritto e sulla legislazione (p. 273).

Per quanto non usi il termine, si potrebbe quindi dire che Bentham

${ }^{15}$ Cfr. J. Bentham, The Theory of Legislation, edited and with an Introduction by C.K. Ogden, London, Kegan Paul, 1931. 
fu, per Oakeshott, un precursore dello 'scientismo' in quanto pensava che la scienza avrebbe liberato l'uomo dalla superstizione e creato una "scienza del governo" che avrebbe applicato "il metodo scientifico al diritto, unito legge e scienza, scoperto qualche mezzo per misurare accuratamente le soddisfazioni politiche" (p. 274). Ma se queste erano le sue ambizioni, sintetizzabili nel credere che nell'ambito delle scienze sociali fosse necessario ripartire da zero ed elaborare un codice: "qualcosa di creato, organizzato e definito, e perciò distinto da ciò che era invece meramente cresciuto" (p. 274), Bentham non aveva le risorse intellettuali per portare a compimento un simile radicale ed originale progetto. Come quei "despoti benevolenti" che furono i philosophes, rimase così a metà strada e il suo odio verso la Common Law gli impedì di capire che anche "la legge deve cambiare" (p. 275). E questo avvenne perché in lui non c'era niente di originale e quindi, in sostanza, perché aveva anche scarsa attitudine per la filosofia. Tant'è che anche riguardo a quell'utilitarismo di cui viene considerato il moderno rifondatore, il suo pensiero diventa confuso e contraddittorio allorché, per quanto riguarda il concetto di utilità, si "avventura oltre ciò che altri avevano pensato" (p. 281). E questo perché quantunque avesse per tutta la vita parlato di "princìpi primi", non avendo un vero interesse per il pensiero speculativo, Bentham "non riuscì mai a considerare con attenzione altro che cose secondarie e derivate" (p. 284).

Come dire che se la filosofia è tutto sommato una pratica innocente ed inutile, un diletto per gentiluomini, una mezza filosofia è invece qualcosa che in genere è destinata a rivelarsi dannosa.

\title{
OAKESHOTT E IL RAZIONALISMO IN POLITICA
}

\author{
di Giovanni Giorgini*
}

L'attacco al razionalismo in politica costituisce il fulcro degli interessi teorici, e delle preoccupazioni pratiche, di Oakeshott negli anni Cinquanta. Con il termine razionalismo egli intende il supposto uso

* Università di Bologna. 